\title{
Retrocausality and quantum mechanics
}

\author{
David T. Pegg* \\ Centre for Quantum Computer Technology, School of Biomolecular and Physical \\ Sciences, Griffith University, Nathan, Brisbane, 4111, Australia
}

\begin{abstract}
The results of experiments in quantum mechanics can be predicted correctly either by assigning a forward-evolving state to the system based on the preparation outcome or by assigning a state that evolves backwards in time based on the measurement outcome. The latter picture admits some retrocausality without allowing messages to be sent at a faster speed than that of light. This retrocausality allows some standard quantum paradoxes to be examined from a different viewpoint. It also allows closed causal cycles to be examined in the context of laboratory experiments. For a particular experiment, we find agreement with the principle that inconsistent causal loops have zero probability of occurring, that is, only self-consistent loops can occur.
\end{abstract}

Keywords: Quantum mechanics; Retrocausality; Causal loops; Time paradox

*E-mail address: D.Pegg@griffith.edu.au 


\section{Introduction}

The notion of causality has long been associated with the concept of an arrow of time: the effect of an event can only be felt after the event, that is, a cause precedes its effect. In a retrocausal situation the effects of an event are felt before the event. Some authors divide causality into two principles: the weak and the strong (Cramer, 1980). The weak causality principle states that a controllable message cannot be sent backwards in time in any reference frame, that is, classical information cannot be transmitted faster than light. Strong, or strict, causality is somewhat less precise but effectively says that cause must always precede effect even at a microscopic level. The weak principle follows from the strong but not vice versa, that is, it is possible for the weak principle to hold while the strong principle is violated. In quantum mechanics there is experimental evidence for only the weak principle.

The strong principle of causality is manifest in quantum mechanics in the assignment of a state to the quantum system. The basic quantum experiment is one in which a system is prepared at some time, allowed to evolve and is then measured at a later time. A fundamental question is how we describe the system between the preparation and measurement events, that is, how we ascribe a state to the system in this interval. In deterministic classical physics, the choice is restricted. Consider a simple experiment in which we prepare a gyroscope to point in a particular direction, say the positive $z$-direction. We then measure the direction of the gyroscope at a later time but after an interval shorter than that needed for the gyroscope to change its direction significantly due to external influences. The result of a good measurement will show that 
the gyroscope is pointing in the positive $z$-direction. The state that we should assign to the system between preparation and measurement is therefore unambiguous: the positive $z$-direction correlates with both the preparation event and the measurement event.

If we substitute a spin-half particle for the gyroscope, however, we have a very different situation. If the particle is prepared with its spin in the positive $z$-direction then we have a $50 \%$ chance of measuring it to be in the positive $x$-direction if we choose the appropriate measuring device. If this occurs, which state do we assign to the particle between preparation and measurement - the state in which we prepared it or the state we measured it to be in? Traditionally we assign the prepared state. The conventional picture is of the spin being in the positive $z$-direction and then suddenly changing to the positive $x$-direction at the time of the measurement. This is based on our notion of strong causality. We feel that the state should be influenced by the earlier preparation event and not by the later measurement event. If the measurement could influence the state at an earlier time then, even though we would have no control over whether the spin is in the positive or negative $x$-direction, our ability to choose the measuring apparatus itself would give us some control over the earlier state. For example we could choose either to use the above measuring device or one that would put the spin in the positive or negative $y$-direction. This would give us control over whether the earlier state is one of the $x$ states of spin or one of the $y$-states. It seems much safer to protect causality by assigning the state on the basis of the preparation event.

In this paper we examine the alternative: assigning the state on the basis of the measurement event and allowing it to evolve backwards in time to the preparation event. This means that it is the outcome of the measurement, rather than of the preparation 
procedure, that determines the state of the system between preparation and measurement. We shall see that this form of retro-evolution, while clearly at odds with strong causality, does not necessarily violate weak causality and can be useful in giving a different viewpoint for the consideration of some well-known quantum effects. We also examine in this paper an even more interesting picture than either the fully forward-evolving or fully retro-evolving interpretations. The use of some retro-evolution in conjunction with forward time evolution in experiments involving more than one measurement event opens the possibility of closed causal cycles, which in turn can lead to well-known time travel paradoxes such as the grandfather paradox. We show in this paper how to set up a particular experiment containing such a causal cycle. We find that in this experimental arrangement only self-consistent cycles are allowed with the probability of inconsistent cycles being zero. This is in accord with the principle of self-consistency.

\section{Retro-evolving quantum states}

Because of the non-deterministic nature of measurement outcomes, the main use of quantum mechanics is to calculate probabilities. A general measurement postulate (Helstrom, 1976) is that the probability of a measurement outcome $j$ given a preparation event $i$ when the time between preparation and measurement is much shorter that the characteristic evolution time of the system is given by

$$
P(j \mid i)=\operatorname{Tr}\left(\hat{\rho}_{i} \hat{\Pi}_{j}\right) .
$$

Here $\operatorname{Tr}$ is the trace, $\hat{\rho}_{i}$ and $\hat{\Pi}_{j}$ are operators acting on the Hilbert space of the system that are associated with the preparation and measurement outcomes respectively. Both are non-negative definite but have different normalization conditions: the trace of $\hat{\rho}_{i}$ is 
unity and the sum of $\hat{\Pi}_{j}$ over all possible measurement outcomes $j$ is the unit operator. $\hat{\rho}_{i}$ is called the density operator and $\hat{\Pi}_{j}$ for all possible $j$ form the elements of a probability operator measure (POM). Provided the normalization conditions hold, these can be quite general non-negative operators. Special cases of both operators are pure state projectors. Although equation (1) is often taken as the fundamental measurement postulate of quantum mechanics, for example as described by Helstrom (1976), it can be derived from a more fundamental postulate that is symmetric in preparation and measurement. The details of this derivation are given by Pegg, Barnett \& Jeffers (2002). The imposition of weak causality removes this symmetry, with weak causality being ensured by the above asymmetry in the normalization conditions (for a detailed explanation of this, see Pegg \& Pregnell, 2004, Pregnell, 2004, and Pegg, 2006).

A more general situation is where the interval between the preparation time $t_{i}$ and the later measurement time $t_{j}$ is long enough for significant evolution to take place. This is taken into account in the standard formalism of quantum mechanics by modifying equation (1) to become, for a closed system,

$$
P(j \mid i)=\operatorname{Tr}\left[\hat{U}\left(t_{j}, t_{i}\right) \hat{\rho}_{i} \hat{U}^{\dagger}\left(t_{j}, t_{i}\right) \hat{\Pi}_{j}\right] .
$$

Here $\hat{U}\left(t_{j}, t_{i}\right)$ is the unitary time-displacement operator governed by Schrödinger's time evolution equation (Merzbacher, 1998). The unitary transformation preserves the unit trace of $\hat{\rho}_{i}$ so the normalisation conditions ensuring weak causality still apply. A simple and direct interpretation associated with (2) is that the preparation event determines the state of the system between $t_{i}$ and $t_{j}$. In this interpretation, the prepared state of the system, as represented by the density operator $\hat{\rho}_{i}$, evolves forward in time from $t_{i}$ to the 
later time $t_{j}$ at which time it may experience a sudden change caused by the

measurement. As this interpretation is accord with our notion of strong causality described above, it appears at first sight that using Schrödinger's equation to extend equation (1) to equation (2) both preserves weak causality and introduces strong causality. Experimental verification of (2) might then be taken as evidence of the need for strong causality.

The cyclic nature of the trace, however, allows us to rewrite (2) equivalently as

$$
\begin{aligned}
P(j \mid i) & =\operatorname{Tr}\left[\hat{\rho}_{i} \hat{U}^{\dagger}\left(t_{j}, t_{i}\right) \hat{\Pi}_{j} \hat{U}\left(t_{j}, t_{i}\right)\right] \\
& =\operatorname{Tr}\left[\hat{\rho}_{i} \hat{U}\left(t_{i}, t_{j}\right) \hat{\Pi}_{j} \hat{U}^{\dagger}\left(t_{i}, t_{j}\right)\right]
\end{aligned}
$$

with the second line following from the unitary nature of time-displacement operator (Merzbacher, 1998). A simple and direct interpretation associated with (3) is that the measurement event determines the state of the system between $t_{i}$ and $t_{j}$ : the measured state $\hat{\Pi}_{j}$ evolves from the measurement time $t_{j}$ to the earlier time $t_{i}$, that is, it evolves backwards in time. We refer to this, for convenience, as retro-evolution. In this interpretation the sudden change from the prepared state to the (retro-evolved) measured state occurs immediately following the preparation process at $t_{i}$ with the state after this change being $\hat{U}\left(t_{i}, t_{j}\right) \hat{\Pi}_{j} \hat{U}^{\dagger}\left(t_{i}, t_{j}\right)$, which is determined by $\hat{\Pi}_{j}$. The unitary transformation preserves the unit sum of the elements $\hat{\Pi}_{j}$ so the normalisation conditions ensuring weak causality still apply. (We note here that we are referring to the POM element $\hat{\Pi}_{j}$ as a state. If we wish to reserve this term for a normalized state, that is, a density operator, then we can easily convert $\hat{\Pi}_{j}$ to a state by dividing by its trace. There 
is no need to do this, of course, when $\hat{\Pi}_{j}$ is a projector as it is already a normalized state.) Although weak causality still applies, our idea of strong causality does not fit in with the retro-evolution of the state and will need to be re-examined. Since with retroevolution the sudden change of state occurs immediately after the preparation process, it is tempting to try to preserve strong causality by saying that it is the preparation and not the measurement that causes the change. This does not really help, however, as the states $\hat{U}\left(t_{i}, t_{j}\right) \hat{\Pi}_{j} \hat{U}^{\dagger}\left(t_{i}, t_{j}\right)$ to which the change can be made depend on the choice of the measurement apparatus, which can be made at a later time. Also, as seen below, the change can be considered to occur at any time between preparation and measurement. We have assumed in the above discussion that the system is closed in order to find the retro-evolving measured state. This assumption is not essential. For an open system the evolution is not unitary, instead the prepared state has a trace-preserving evolution governed by a master equation. We can again write the probability in terms of a retro-evolving state that preserves the unit sum of the POM elements $\hat{\Pi}_{j}$ (Barnett, Pegg, Jeffers \& Jedrkiewicz, 2001). However we shall not discuss this more complicated system here, as the simpler closed system is sufficient to display the nature of retroevolution.

Expression (3) is not the only way of rewriting (2). The group property of the time evolution operator allows us to write in general

$$
\hat{U}\left(t_{j}, t_{i}\right)=\hat{U}\left(t_{j}, t\right) \hat{U}\left(t, t_{i}\right)
$$

where $t$ is any time between preparation and measurement. From this we can write

$$
P(j \mid i)=\operatorname{Tr}\left[\hat{U}\left(t, t_{i}\right) \hat{\rho}_{i} \hat{U}^{\dagger}\left(t, t_{i}\right) \hat{U}\left(t, t_{j}\right) \hat{\Pi}_{j} \hat{U}^{\dagger}\left(t, t_{j}\right)\right]
$$


This expression incorporates the forward evolution of $\hat{\rho}_{i}$ from $t_{i}$ to $t$ and the retro-

evolution of $\hat{\Pi}_{j}$ from the measurement time back to $t$. We see from this that we can say that the sudden change of state, or collapse, occurs at any time $t$ between preparation and measurement. Clearly (2) and (3) are special cases of (5) determined by choosing $t$ to be $t_{j}$ or $t_{i}$. Can we still retain the quantum mechanical notion that the act of measurement at time $t_{j}$ induces, or causes, the discontinuous change of state? We can retain this notion, and it is useful to do so, if we accept the idea of retrocausality associated with retro-evolution. It is important to note that this form of retrocausality violates only strong causality. The transformations of $\hat{\rho}_{i}$ and $\hat{\Pi}_{j}$ in (5) do not change the normalization conditions that ensure weak causality.

\section{Schrödinger's cat}

The expressions (2), (3) and (5) are mathematically equivalent and thus are all compatible with the standard mathematical formalism of quantum mechanics, that is, they all yield precisely the same probabilities for the outcomes of measurements, even though they are associated with very different physical interpretations. For our first example, we look at the retrocausal interpretation associated with expression (3) and the different viewpoint it may give in examining the well-known Schrödinger's cat paradox. In the Schrödinger's cat thought experiment, a cat inside a box is prepared in a state $\hat{\rho}_{i}$ that is a superposition of being dead and being alive. After some time, for example one hour, the box is opened and an observation, or measurement, is made which is designed to determine if the cat is dead or if it is alive. We represent these two possible outcomes by the POM elements $\hat{\Pi}_{d}$ and $\hat{\Pi}_{a}$ respectively. A measurement procedure involving 
simply looking at the cat is sufficient to achieve these outcomes. (Presumably more sophisticated measurement techniques would be needed if, for example, we wanted the

measurement POM elements to be $\hat{\Pi}_{+}$and $\hat{\Pi}_{-}$, representing the "alive plus dead" and the "alive minus dead" states. We shall not pursue this here, however, as we are only interested in the standard paradox situation.) In the standard situation, the superposition state $\hat{\rho}_{i}$ evolves in the forward time direction until the observation. If the cat is observed to be dead, say, the superposition state immediately changes to the dead state and the cat is finally killed at the time of the measurement. If we adopt the full retro-evolution picture, on the other hand, the dead state $\hat{\Pi}_{d}$ associated with the outcome of the measurement evolves backwards in time until the time of the preparation, when the cat is killed by the preparation device. In this picture the cat is dead all the time between preparation and measurement, that is, it is not in a superposition or "limbo" state at any time, which is more in keeping with a classical picture involving macroscopic objects. Accepting retrocausality in the sense that the choice of an observation procedure with

POM elements $\hat{\Pi}_{d}$ and $\hat{\Pi}_{a}$ determines the possible states of the cat at an earlier time thus allows us to remove the paradox of the superposition state if we so wish. Weak causality, of course, still holds.

\section{EPR experiments}

Early experiments verifying the Einstein-Podolsky-Rosen (EPR) paradox (Einstein, Podolsky \& Rosen, 1935) involved photon pairs from an atom decaying from a $J=0$ excited state to a $J=0$ ground state via a degenerate $J=1$ intermediate state. Photodetectors, with polarization analysers in front of them, were in modes $b$ and $c$ on 
opposite sides of the atom (Kocher \& Commins, 1967; Freedman \& Clauser, 1972; Aspect, Dalibard \& Roger, 1982). This allows two photons to be detected with correlated polarizations. In the traditional interpretation, an entangled state of the two photons propagates forwards in time from the atom until it is collapsed to a one-photon state by a detection event. This two-photon entangled state can be written in terms of linear polarized photons as (Clauser, 1972)

$$
2^{-1 / 2}\left(|H\rangle_{b}|H\rangle_{c}+|V\rangle_{b}|V\rangle_{c}\right)
$$

where the subscripts denote the two modes $b$ and $c$ and $H$ and $V$ refer to horizontal and vertical polarizations. From the observed correlations it appears that the setting of the analyser at one detector immediately affects the polarization of the other photon, which is a sizeable distance away, in violation of the concept of Einstein separability. For example, suppose the analyser in mode $b$ is set so as to allow horizontally polarised light to pass through. Then the observed correlations are consistent with the detection of a photon by the detector B in mode $b$ collapsing the entangled field state (6) to the projection of the measured state $|H\rangle_{b}$ onto the state (6) in accord with quantum measurement theory. That is, the correlations are consistent with the detection event at B causing the state of the other photon immediately to become $|H\rangle_{c}$. The results of the experiments do not seem to be consistent with the retention of all the notions of objective reality, locality and causality. Often objective reality is considered to be the most likely candidate for rejection. It is worth, however, considering alternative interpretations that can arise from abandoning strong causality and just retaining weak causality. This allows us to incorporate some retro-evolution. As there are two measurements in the above experiment, we need to choose the times at which the sudden change of state, or collapse, 
associated with each of these measurements takes place. In the description below we choose these times to give retro-evolution for the first photon emitted, that is, the photon in mode $b$, and forward evolution for the photon in mode $c$.

Let the excited, ground and intermediate states of the atom be $|e\rangle_{a},|g\rangle_{a}$ and $|m\rangle_{a}$ respectively where $m$ can take the values 1,0 and -1 , and let the first photon emitted be in mode $b$ to the right of the atom. We write the initial atom-field state as $|e\rangle_{a}|0\rangle_{b}|0\rangle_{c}$ indicating that there are no photons in the two detector modes. Then, after a very short time such that there is negligible probability for the atom to be in the ground state, the initial atom-field state will have evolved to an entangled state that is a superposition of the initial state and a state $|I\rangle_{a b}|0\rangle_{c}$ where $|I\rangle_{a b}$ is a superposition of states $|m\rangle_{a}|p\rangle_{b}$, where $|p\rangle_{b}$ is a one-photon state in mode $b$ with a polarization $p$. For each value of $m$ in the superposition there is a corresponding polarization $p$. By choosing the axis of quantization $z$ in the direction of detector B in mode $b$, we find that we can write the state $|I\rangle_{a b}$ explicitly in the linear polarization basis as

$$
\left.|I\rangle_{a b}=1 / 2\left[|+1\rangle_{a}\left(-|H\rangle_{b}+i|V\rangle_{b}\right)+|-1\rangle_{a}(H\rangle_{b}+i|V\rangle_{b}\right)\right]
$$

where \pm 1 are values of $m$ and $|H\rangle_{b}$ and $|V\rangle_{b}$ are single photon states with polarizations in the horizontal and vertical directions. The superpositions of $|H\rangle_{b}$ and $|V\rangle_{b}$ in (7) are single photon states with circular polarizations.

Let the POM elements for the detector B in mode $b$ be $\hat{\Pi}_{y}(q)$ and $\hat{\Pi}_{n}(q)$ where the former represents a click, signifying that a photon with polarization $q$, which is the polarization of the analyser, has been counted. The latter represents the no-click 
outcome. In the event that there is a click, we can follow the retro-evolution of the corresponding one-photon state with polarization $q$ back to the atom, at which time the atom-field entangled state collapses to the projection of this retro-evolved one-photon state onto the atom-field entangled state. Assuming that the medium is such that the polarization $q$ is not altered during the retro-evolution, the resulting collapsed atom-field state is ${ }_{b}\langle q \mid I\rangle_{a b}|0\rangle_{c}$. The state ${ }_{b}\langle q \mid I\rangle_{a b}$ will be a superposition of the states $|m\rangle_{a}$ with the coefficients determined by the values of ${ }_{b}\langle q \mid p\rangle_{b}$. This superposition will determine the polarization of the second photon emitted, which clearly will be correlated with $q$. For example, if a horizontally polarized photon is detected by detector B the collapsed intermediate state of the atom is ${ }_{b}\langle H \mid I\rangle_{a b}$, which from (7) is $2^{-1 / 2}\left(|-1\rangle_{a}-|+1\rangle_{a}\right)$, and the transition from this state to the ground state produces a horizontally polarized photon $|H\rangle_{c}$. We can then follow the propagation of the second photon forwards in time and calculate the probability of it passing through the analyser at the detector $\mathrm{C}$ in mode $c$ on the left of the atom. This probability will depend on $q$, that is, on the setting of the analyser at the detector B. This is still the case even when the detector B and its analyser are at a much greater distance from the atom than the detector $\mathrm{C}$ and its analyser so that the detection of the second photon emitted takes place before the detection of the first photon and even before the setting of the analyser at B has been decided.

In the above description of the experiment, which uses retro-evolution of the first photon state, which we might describe as retro-propagation of the first photon, and normal propagation of the second photon, the change of state associated with the measurement of the first photon occurs locally, that is, at the atom. This local collapse is 
exactly what would happen if the detector B were placed at the atom itself, allowing the measurement of the photon to collapse the atom-field state immediately upon detection before the second photon is emitted. The paradox with Einstein separability is avoided. The price paid for this is, of course, the abandonment of strong causality by the adoption of retro-evolution. Weak causality is still preserved and one observer, Bob, cannot make use of the violation of strong causality to send a message to Alice backwards in time or even at a faster speed than that of light. To see this, suppose Bob, who has control over the analyser setting at the detector B wishes to send a message to Alice at the detector $\mathrm{C} \mathrm{a}$ large distance away. Bob knows the polarization setting of Alice's analyser and so can choose the setting of his own analyser such that, in the event that he detects a photocount, he knows that Alice cannot detect a count. Alternatively he can choose his setting such that, in the event that he detects a photocount, he knows that Alice can detect a count. He hopes by this means to send a message in a suitable code. What prevents him from sending any controllable information is that, although he controls his analyser setting, he has no control over whether his detector counts the photon or whether it is absorbed by his analyser. In the latter case the retro-propagating field, and thus the second photon, will have a polarization state orthogonal to that for the former case. Thus the photocount that Alice records could correspond either to a particular setting of Bob's analyser and a photocount registered by Bob or to the orthogonal setting and first photon being absorbed by the Bob's analyser rather than his detector. Alice would be able to decide which is the case if Bob could tell her whether he recorded a count or not but this information would not reach Alice until a later time. 


\section{Causal loops}

In describing the EPR-type experiment, we invoked both retrocausality and normal causality for different parts of the process. If both types of causality are allowed in the process then it should be possible to form a causal loop, that is, a closed causal cycle of events. Such loops have been associated with the possibility of time travel into the past, giving rise to paradoxes of which the most well known is the grandfather paradox. The essence of the grandfather paradox is that if a person can have a retrocausal influence over the circumstances leading to his or her birth, then he or she can prevent his or her own birth. The classic example is a time traveller who goes back in time and kills his or her grandfather well before the grandfather has children, in which case the time traveller is not born and so does not kill the grandfather, in which case the time traveller is born and so on. The grandfather paradox is a generic term including the case of autoinfanticide, in which the time traveller simply shoots himself or herself as a baby. Of course the retrocausal influence need not be consciously controlled, a paradox will arise even if simply the existence of the person or object is arranged to lead retrocausally to its non-existence or sufficiently early demise.

Sometimes the possibility of such paradoxes is taken as a reason to disallow causal loops and thus the possibility of time travel. An alternative argument invokes the principle of self-consistency as a means of resolving paradoxes (see, for example, Wheeler \& Feynman, 1949; Schulman, 1971; Peres \& Schulman, 1972; Novikov, 1998). According to this principle, causal loops are allowed provided they are self-consistent, which would allow time travel but with some restrictions. An example of a consistent loop is one in which a time traveller goes into the past and ensures that his father meets 
his mother, thereby ensuring his own birth. In these particular examples of classical causal loops the retrocausality involved violates weak causality. However it should also be possible to set up causal loops in quantum mechanical experiments in which the retrocausality only violates strong causality, in which case such loops may be accessible in the laboratory. Here we look at modifying an EPR type of experiment to do this.

In the EPR type of experiment, as we have described it in terms of retrocausality and normal causality, the causal pattern in space-time is V-shaped if we take the time axis as pointing upwards. This is shown in Fig. 1. The photon detection event at detector B is at the top right part of the $\mathrm{V}$, the atom emission events at the bottom of the $\mathrm{V}$ and the detection event at detector $\mathrm{C}$ at the top left. The causal direction points down along the right of the $\mathrm{V}$ and up along the left. To form a loop we need to close the top of the $\mathrm{V}$. We can do this as shown in Fig. 2. Here we replace detector B and its analyser by a suitable reflector that reflects the light path of mode $b$ back so that it is approximately parallel to mode $c$ but such that it misses the atom and detector C. For simplicity, let us assume that this is done in a way that does not change the polarization of the field in mode $b$. Detector B with its analyser is placed in the reflected mode $b$ somewhere near detector $\mathrm{C}$. The geometry is chosen so that the total optical path length from the atom to detector $\mathrm{B}$ is much greater than the path length from the atom to detector $\mathrm{C}$, allowing sufficient time for information about the detection event at $\mathrm{C}$ to be sent and used to adjust the analyser at B before the other detection event takes place at detector B. In terms of our retrocausal description the loop is now complete.

Let the above closed loop apparatus be arranged so that initially the linear polarization of the analysers at detectors $\mathrm{B}$ and $\mathrm{C}$ are set to allow horizontally polarized 
light to pass through and a detection event at $\mathrm{C}$ is set to trigger a shift in the polarization of the analyser B by an angle $\theta$. We can describe a feedback mechanism from the future to the past in terms of a retro-propagating photon in mode $b$ as follows. As described in the previous section, if detector $\mathrm{B}$ registers a photocount, the photon in mode $b$ retropropagates backwards in time from the detector with the same polarization as the analyser at B to reach the atom prior to the emission of the photon in mode $c$. This collapses the atom-field state to an intermediate atomic state correlated with the polarization of the analyser at $\mathrm{B}$, which in turn causes the photon in mode $c$ to be emitted from the atom with the same polarization as the analyser at B. If, on the other hand, detector $\mathrm{B}$ does not register a photocount, indicating that the photon in mode $b$ is absorbed by the analyser at $\mathrm{B}$, the retro-propagating photon has a polarization orthogonal to that of the analyser at B and thus causes the photon in mode $c$ to have a polarization orthogonal to the polarization of this analyser.

Now consider the life of the photon in mode $c$, which we describe in the normal time direction. After the photon in mode $c$ is "born", that is, emitted by the atom, it propagates forwards in time with some polarization until it encounters the analyser in mode $c$. If it is absorbed by this analyser, it "dies" before it has any chance of reaching detector $\mathrm{C}$ and influencing its birth by means of the feedback mechanism described above. If it passes through this analyser, however, it is detected by the detector C. This causes the analyser at $\mathrm{B}$ to be rotated through an angle $\theta$. This in turn causes the retropropagating photon in mode $b$ to have a polarization at either $\theta$ or $\theta+\pi / 2$ to the horizontal, depending on whether detector B does or does not register a photocount, 
causing the photon in mode $c$ to be emitted with a polarization at either $\theta$ or $\theta+\pi / 2$ to the horizontal.

Consider the scenario in which the detector B does register a photocount. In this case we can model the grandfather type of paradox as follows. We choose the angle $\theta$ to be $\pi / 2$, representing the maximum change possible. Then the survival of the photon in mode $c$ that allows it to reach detector $\mathrm{C}$ causes the retro-propagating photon in mode $b$ to have a vertical polarization. This causes the photon in mode $c$ also to have a vertical polarization, causing it to be absorbed by the analyser in mode $c$, that is, to die before it reaches the detector $\mathrm{C}$. Thus the analyser at $\mathrm{B}$ is not altered, which means that the retropropagating photon has a horizontal polarization, which in turn gives the photon in mode $c$ a horizontal polarization that causes it to survive the analyser and so on. We can either take this as an argument against the retrocausality involved or retain retrocausality and see there is a reason why the principle of self-consistency should be applicable here. According to this principle, such a paradoxical causal loop should not be able to occur, that is, the probability for it to happen should be zero. The paradox scenario is based on detector B registering a count and so the probability of it occurring will be proportional to the probability that detector B registers a count. There are four possible joint outcomes of the ideal experiment in which two photons are emitted. Two of these include a count at detector B: a count at B with a count at detector $\mathrm{C}$ and a count at $\mathrm{B}$ with no count at $\mathrm{C}$. From our earlier discussion of the mathematical equivalence of the different pictures described in this paper, the calculated probabilities of these measurement outcomes are independent of whether we use the conventionally evolving state picture or a retroevolving state picture to calculate them. It is more straightforward to perform this 
calculation by making use of the former description. In this description, at a time just before the photon in mode $c$ encounters its analyser, the two photons are in the entangled state given by expression (6). If the photon in mode $c$ passes through this analyser and is thus detected, the state of the photon in mode $b$ is simultaneously collapsed to $|H\rangle_{b}$, that is, it acquires a horizontal polarization. Before the photon in mode $b$ reaches its analyser, however, the detection of the photon in mode $c$ rotates the polarization of this analyser to the vertical, with the result that the photon in mode $b$ does not reach its detector. On the other hand, if the photon in mode $c$ is absorbed by its analyser, the state of the photon in mode $b$ is simultaneously collapsed to $|V\rangle_{b}$, that is, it acquires a vertical polarization. In this case, because the photon in mode $c$ does not reach its detector, the polarization of the analyser in mode $b$ remains horizontal, which will also prevent the photon in mode $b$ from reaching its detector. Thus the total probability for detector B registering a photocount is zero. This means that the inconsistent cycle described above never occurs. By suitable choice of initial polarizations of the analysers, inconsistent causal cycles can also be constructed on the premise that detector B does not register a photocount. We find that for these configurations, the probability of this premise being realised is also zero.

We see from the above that even if we do associate retrocausality with retropropagating states, the overall physics is such as to make the probability of the occurrence of an inconsistent causal cycle zero. It is worth also looking at consistent cycles, which also exist for such situations. In the autoinfanticide case, we can find such consistent solutions by ensuring that there is an appropriate correlation between the state of the time traveller and the state of the infant after their encounter. An example of such 
a consistent cycle, which has been attributed to Feynman (Pegg, 2001), is as follows: after finding his infant self, the time traveller raises his rifle and takes aim but, because of his bad shoulder, he misses the infant's heart and wounds it in the shoulder. In this case the consistent solution involves a state that deviates from the state of being alive and perfectly functioning but is not the dead state.

We can modify our above experiment for the scenario in which the detector B does register a photocount and find a consistent cycle by allowing the angle $\theta$ to deviate from $\pi / 2$. Then the survival of the photon in mode $c$ that allows it to reach detector $\mathrm{C}$ to be detected will cause the analyser at $\mathrm{B}$ to have a polarization at angle $\theta$ to the horizontal, so the retro-propagating photon will have this polarization and so too will the photon in mode $c$. Thus the latter photon has a chance given by $\cos ^{2} \theta$ of passing through its analyser and being detected at detector $\mathrm{C}$, thereby completing the consistent causal cycle. Evidence of this consistent loop occurring is the detection of photons both at detector B and detector C. We can calculate the probability that, of the four possible experimental outcomes of the ideal experiment in which two photons are emitted, this is the one that occurs and again we do this by using the conventional forward evolving entangled state description. We find this probability, which is normalized so that the total probability of obtaining any one of the four possible measurement outcomes is unity, to be $\left(\cos ^{2} \theta\right) / 2$. Thus when $\theta=\pi / 2$ there is no possibility of a consistent loop with photocounts at both detectors, which is the case we studied previously. If $\theta \neq \pi / 2 \mathrm{a}$ consistent loop is possible. For this case both the forward and retro-propagating photons have a polarization that deviates from the horizontal and vertical polarizations involved in the previous $\theta=\pi / 2$ case. 
For the $\theta \neq \pi / 2$ case, in addition to the causal loop above, there are three other loops associated with the other three experimental joint outcomes of the ideal experiment: no photocounts at detector $\mathrm{C}$ with a photocount at detector $\mathrm{B}$; no photocounts at detector $\mathrm{C}$ with no photocounts at detector $\mathrm{B}$; and a photocount at detector $\mathrm{C}$ with no photocounts at detector B. The normalized probabilities for these outcomes to occur are, respectively, $0,1 / 2$ and $\left(\sin ^{2} \theta\right) / 2$. In the first two of these cases there is no photocount at detector $\mathrm{C}$ so the analyser in mode $b$ is not altered. The probabilities of these occurring are therefore just the same as for the normal EPR experiment with no feedback to alter the original horizontal analyser settings, in which case a photocount in detector B must be associated with a photocount in detector $\mathrm{C}$. Thus the probability of no photocount at detector $\mathrm{C}$ with a photocount at detector B is zero. The probability of no photocounts in both detectors is $1 / 2$ after the normalization described above. We can construct causal loops in a similar manner to what we have done above, that is, by starting with the detection or non-detection of photon $c$ at detector $\mathrm{C}$ with its effect on the analyser at $\mathrm{B}$ and then tracing the causal path by means of the retro-propagating photon in mode $b$ back to the atom. We note that for no photocount in detector B, that is, for absorption by the analyser at B, the retro-propagation is from the analyser and the polarization of the retropropagating photon is orthogonal to that of the analyser. When we construct these causal loops, we find that for the first of the three joint outcomes above, that is no count in $\mathrm{C}$ but one in B, the loop formed is inconsistent. For the second of these joint outcomes, in which no photocounts are detected, the loop formed is consistent. In this case the mode $b$ photon is vertically polarised and so too will be the mode $c$ photon, which will therefore be absorbed by the analyser in mode $c$. For the third joint outcome, a count in 
C but none in $\mathrm{B}$, a consistent loop can occur with a chance of $\sin ^{2} \theta$, which is the probability that the mode $c$ photon will reach the detector $\mathrm{C}$ to complete the loop consistently. To sum up the four cases described, the probabilities of the associated joint outcomes are, in the order given above, $\left(\cos ^{2} \theta\right) / 2,0,1 / 2$ and $\left(\sin ^{2} \theta\right) / 2$ where these are normalized to sum to unity. The corresponding probabilities for a consistent loop to be

formed in each case are $\cos ^{2} \theta, 0,1$ and $\sin ^{2} \theta$. These results indicate that the probability for a loop to occur is proportional to the probability that it is consistent.

All the results of this section confirm the principle of self-consistency that physics is such that inconsistent causal cycles cannot happen, that is, their probability is zero. Further, not all consistent cycles are equally likely; there is a smooth transition from the most likely cycles down to ones with vanishing probabilities. These results are consistent with that for the causal loop formed by retro-propagation in an entirely different experimental configuration based on the quantum scissors device (Pegg, Phillips \& Barnett, 1998), for which it was seen that the amplitude for an inconsistent loop effectively cancels itself out (Pegg, 2001). A different type of quantum mechanical loop has also been proposed by Ralph (2005).

\section{Conclusion}

In addition to the conventional interpretation of quantum mechanics in terms of states that evolve forwards in time in accord with strong, or strict, causality, the formalism of quantum mechanics also permits an interpretation in terms of retro-evolving states. The formula for calculating probabilities of measurement outcomes in the latter interpretation is mathematically equivalent to that used in the conventional interpretation. 
Consequently the retro-evolving state interpretation does not change any of the results of experiments predicted by the conventional interpretation, even though the associated retrocausality violates some of our notions of strong causality. Also, this interpretation does not violate the important weak principle of causality that a controllable message cannot be sent faster than light. As the particular retrocausal picture presented in this paper cannot be distinguished from the conventional picture by physical experiments, there is no reason to give either picture any priority. The retrocausal picture does, however provide a different viewpoint. It allows an interpretation of the Schrödinger cat thought experiment more in keeping with our classical ideas, if that is considered desirable.

In this paper a picture is also constructed that contains elements of both strong causality and retrocausality, but which does not violate weak causality, by involving both forward evolving and retro-evolving states. This picture predicts the same measurement outcomes as the standard picture for a typical Einstein-Podolsky-Rosen type of experiment but allows us to retain locality and realism at the price of retaining only weak, and not strong, causality. The V-shaped causality pattern in space-time is reminiscent of the absorber theory (Wheeler \& Feynman, 1945) description of such experiments in which the retrocausality is associated with the advanced field from the detector that supplies the radiative reaction causing the downwards transition in the atom (Cramer, 1980; Pegg, 1980). There is, however, a very important difference between absorber theory and the quantum mechanical picture involving both forward evolving and retroevolving states discussed in this paper. Absorber theory is a different theory from standard electrodynamics, in which the radiative reaction arises from the action of the 
charge on itself, and would be experimentally distinguishable from the standard theory in a universe with suitable absorptive properties (Heron \& Pegg, 1974). The picture involving both forward evolving and retro-evolving states, on the other hand, is entirely compatible with the standard mathematical formalism of quantum mechanics and is experimentally indistinguishable from other interpretations, such as the conventional evolutionary interpretation that preserves strong causality but not local realism, that are also accommodated within this formalism.

We have also looked at the consequences of closing the causal cycle in an EPR type of experiment. This allows us to study, in the context of a laboratory experiment, inconsistent causal cycles of the type involved in the time-travel grandfather paradox. We obtain results in accord with the principle of self-consistency that physics is such as to prevent the occurrence of inconsistent cycles. In general we find that the probability of occurrence of a particular cycle is proportional to the probability that it is self-consistent. We should note that we have only arrived at this result for the cycles discussed in this paper, which do not violate weak causality and predict the same measurement probabilities as the standard causal interpretation. We would not expect, for example, cycles that involve the violation of weak causality to predict the same measurement probabilities as a fully causal theory and so this proportionality relation, although reasonable, should not be extended immediately to include such loops.

Overall, while the retrocausal interpretation of quantum mechanics studied in this paper will not predict different experimental results from the conventional interpretation, it may in some circumstances provide an easier way of calculating such results. Further, it provides a different viewpoint from which to examine quantum paradoxes. 


\section{Acknowledgments}

This work has benefited substantially from discussions with Stephen Barnett, John Jeffers and Kenny Pregnell over the years. I thank the Australian Research Council and the Queensland Government for funding.

\section{References}

Aspect, A., Dalibard, J., \& Roger, R. (1982). Experimental tests of Bell's inequalities using time-varying analysers. Physical Review Letters, 49, 1804-1807.

Barnett, S. M., Pegg, D. T., Jeffers, J. \& Jedrkiewicz, O. (2001). Master equation for the retrodiction of quantum communication signals. Physical Review Letters, 86, 2455-2458.

Clauser, J. F. (1972). Experimental limitations to the validity of semiclassical radiation theories. Physical Review A, 6, 49-54.

Cramer, J. G. (1980). Generalized absorber theory and the Einstein-Podolsky-Rosen paradox. Physical Review D, 22, 362-376.

Einstein, A., Podolsky, B. \& Rosen, N. (1935). Can the quantum-mechanical description of physical reality be considered complete? Physical Review, 47, 777-780.

Freedman, S. J. \& Clauser, J. F. (1972). Experimental test of local hidden variable theories. Physical Review Letters, 28, 938-941.

Helstrom, C. W. (1976). Quantum detection and estimation theory. New York: Academic Press.

Heron, M. L. \& Pegg, D. T. (1974). A proposed experiment on absorber theory. Journal 
of Physics A: Mathematical, Nuclear and General, 7, 1965-1969.

Kocher. C. A. \& Commins, E. D. (1967). Polarization correlation of photons emitted in an atomic cascade. Physical Review Letters, 18, 575-577.

Merzbacher, E. (1998). Quantum mechanics (pp. 316-318). New York: Wiley.

Novikov, I. D. (1998). The river of time (p. 254). Cambridge: Cambridge University Press.

Pegg, D. T. (1980). Objective reality, causality and the Aspect experiment. Physics Letters A, 78, 233-234.

Pegg, D. T. (2001). Quantum mechanics and the time travel paradox. In D. Mugnai, A. Ranfagni \& L. S. Schulman (Eds.), Time's arrows, quantum measurement and superluminal behaviour (pp. 113-124). Rome: Consiglio Nazionale delle Richerche. (quant-ph/0506141).

Pegg, D. T. (2006). Causality in quantum mechanics. Physics Letters A 349, 411-414. Pegg, D. T., \& Pregnell, K. L. (2004). Retrodictive state generation and quantum measurement. In S. M. Barnett, E. Anderson, J. Jeffers, P. Ohberg and O. Hirota (Eds.) Quantum communication, measurement and computing (pp. 377-382). New York: American Institute of Physics.

Pegg, D.T., Phillips, L.S. \& Barnett, S.M. (1998). Optical state truncation by projection synthesis. Physical Review Letters, 81, 1604-1606.

Pegg, D.T., Barnett, S.M. \& Jeffers, J. (2002). Quantum theory of preparation and measurement. Journal of Modern Optics, 49, 913-924.

Peres, A. \& Schulman, L. S. (1972). Existence theorem for some differential equations with advanced interactions. Physical Review D, 5, 2654-2655. 
Pregnell, K. L. (2004). Retrodictive quantum state engineering. E-Print Archive: quant-ph/0508088.

Ralph, T. C. (2005). Time displaced entanglement and non-linear quantum evolution. E-Print Archive: quant-ph/0510038.

Schulman, L. S. (1971). Tachyon paradoxes. American Journal of Physics, 39, 481-484.

Wheeler, J. A. \& Feynman, R. P. (1945). Interaction with the absorber as the mechanism of radiation. Reviews of Modern Physics, 17, 157-181.

Wheeler, J. A. \& Feynman, R. P. (1949). Classical electrodynamics in terms of direct interparticle action. Reviews of Modern Physics, 21, 425-433. 


\section{CAPTIONS TO FIGURES}

Fig. 1. A space-time diagram for the EPR-type experiment. The vertical lines, reading from the left, are the world lines of detector $\mathrm{C}$, the analyser for detector $\mathrm{C}$, the atom $a$, the analyser for detector B and detector B. The sloping lines are the world lines of the photons in modes $c$ and $b$. The case illustrated is where a photocount is registered by both detectors, that is, where both the photon world lines reach the detectors.

Fig. 2. Modification to the EPR-type experiment in which the photon in mode $b$ is reflected. The total optical path length for mode $b$, including the reflected part, must be sufficiently long for a signal triggered by a photocount at detector $\mathrm{C}$ to alter the setting of the analyser for detector B before the photon in mode $b$ is at this analyser. 


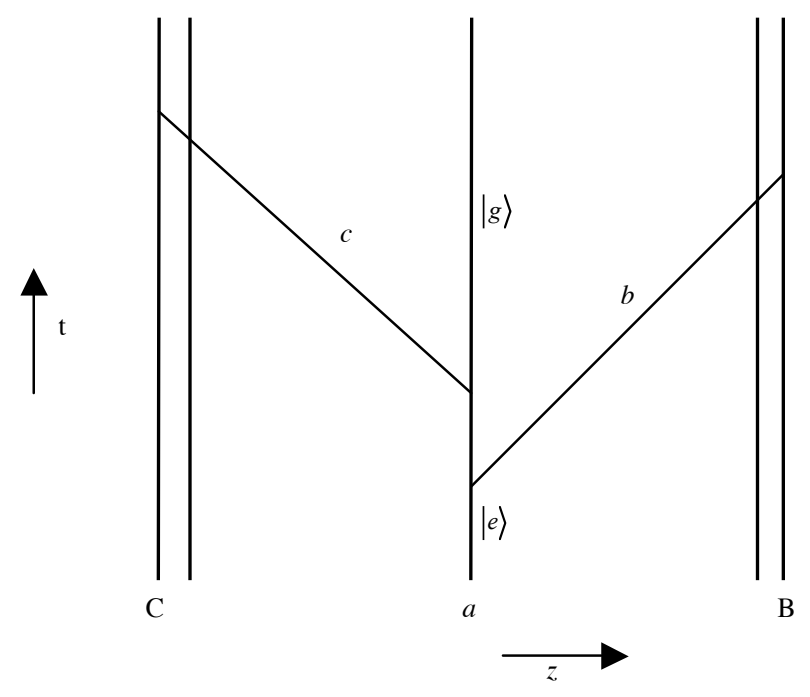

Fig. 1 


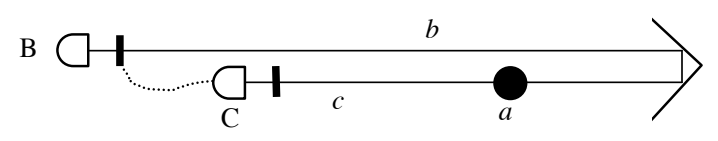

Fig. 2 